\title{
Editorial
}

\section{Metaheuristic Optimization: Algorithmic Design and Applications}

\author{
Gexiang Zhang, ${ }^{1}$ Linqiang Pan, ${ }^{2}$ Ferrante Neri, ${ }^{3}$ Maoguo Gong, ${ }^{4}$ and Alberto Leporati ${ }^{5}$ \\ ${ }^{1}$ School of Electrical Engineering, Southwest Jiaotong University, Chengdu, China \\ ${ }^{2}$ School of Automation, Huazhong University of Science and Technology, Wuhan, China \\ ${ }^{3}$ Centre for Computational Intelligence, School of Computer Science and Informatics, De Montfort University, Leicester, UK \\ ${ }^{4}$ Key Laboratory of Intelligent Perception and Image Understanding, Xidian University, Xian, China \\ ${ }^{5}$ Department of Informatics, Systems and Communication, Università degli Studi di Milano-Bicocca, Milano, Italy
}

Correspondence should be addressed to Gexiang Zhang; gexiangzhang@gmail.com

Received 15 June 2017; Accepted 15 June 2017; Published 5 September 2017

Copyright (C) 2017 Gexiang Zhang et al. This is an open access article distributed under the Creative Commons Attribution License, which permits unrestricted use, distribution, and reproduction in any medium, provided the original work is properly cited.

This special issue focuses on algorithmic design and applications of metaheuristic optimization algorithms. More specifically, we aim at offering some examples of informed design where the problem features are taken into account during the design of the metaheuristic algorithm. Two categories of approaches are here identified:

(i) Algorithms that perform changes during the run on the basis of the success of the components, thus adapting to the problem

(ii) Algorithms that are designed after a thorough problem examination

Some of the papers contained in this special issue mainly focus on algorithmic aspects by modelling and conceptualizing some problem features and/or design approaches. Other papers propose domain specific implementations. However, due to the nature of the subject, the two categories are not clearly separated and, naturally, research in the field tends to lay on both areas.

The paper by E. B. de Moraes Barbosa and E. L. Franca Senne makes use of statistical and artificial intelligence methods to fine-tune the parameters of algorithms. The proposed method, namely, Heuristic Oriented Racing Algorithm (HORA), explores the parameter space to search for a good configuration and thus adapts the algorithm to each problem under consideration.
The paper by S. Alharbi and I. Venkat proposes an evolutionary approach for a problem of chess puzzles, that is, the Minimum Dominating Set of Queens Problem. To tackle this problem, S. Alharbi and I. Venkat propose a simplistic Genetic Algorithm with an encoding and basic algebra especially suited to the problem.

The paper by Y. Lei and J. Shi focuses on a specific class of combinatorial problems, namely, examination timetabling problems. In this paper, the problem is stated as a twoobjective optimization problem and solved by a tailored memetic algorithm. The proposed algorithm uses a previously designed special crossover operator and two local search algorithms combined with a diversity-keeping strategy used to ensure that the nondominated set contains enough solutions.

The paper by Y. Li et al. proposes a novel sophisticated metaheuristic belonging to the class of particle swarm optimizers. The proposed framework is thought to address complex highly multivariate problems and makes use of the MapReduce programming model to decompose the algorithmic operations and allow a natural parallelization.

The paper by $\mathrm{K}$. Li et al. focuses on an image processing problem with a specific reference to agriculture. The proposed system is composed of an evolutionary metaheuristic and two image processing algorithms. The goal of the proposed system is to detect spots on plants due to maize diseases and insect pests. 
The paper by N. Elkhani and R. C. Muniyandi proposes a novel hybridization within multiobjective particle swarm optimization frameworks. This hybridization makes use of a parallelized version of membrane computing to perform the selection within the metaheuristic. The multiobjective particle swarm optimizer is used to identify marker genes for cancer classification.

The last two papers focus on tailored algorithmic design for military applications. The paper by G. Peng et al. proposes a hybrid/memetic discrete particle swarm optimizer to solve a complex real-world military problem. The problem consisting of assigning the targets to the weapons available to maximise the efficiency of an air attack leads to a combinatorial problem which is at the same time multiobjective, dynamic, and constrained. The proposed algorithm is composed of multiple parts including a Cauchy moving operator and local search as well as a repairing algorithm to solve situations with too few feasible solutions.

The paper by K. Zhang et al. studies the unmanned combat aerial vehicle path planning and proposes a new metaheuristic approach which integrates within its structure a domain specific graph theory model. The obtained trajectory of the unmanned aerial vehicle designed by the proposed approach smoothly avoids those areas controlled by radars.

Gexiang Zhang

Linqiang Pan

Ferrante Neri

Maoguo Gong

Alberto Leporati 


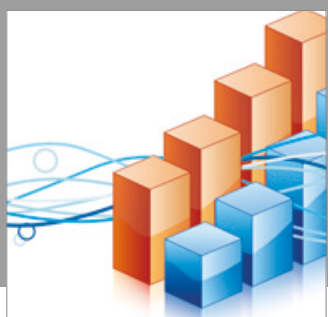

Advances in

Operations Research

vatersals

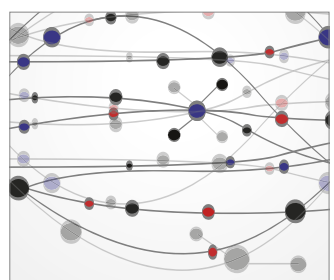

\section{The Scientific} World Journal
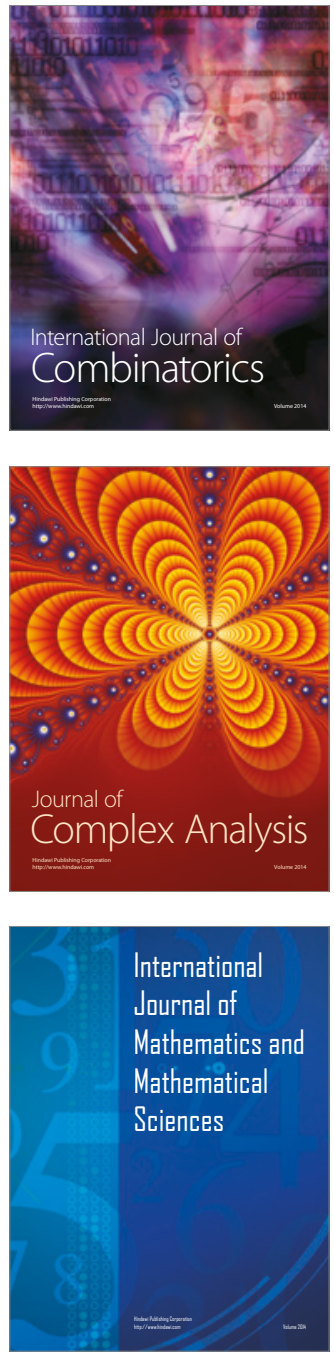
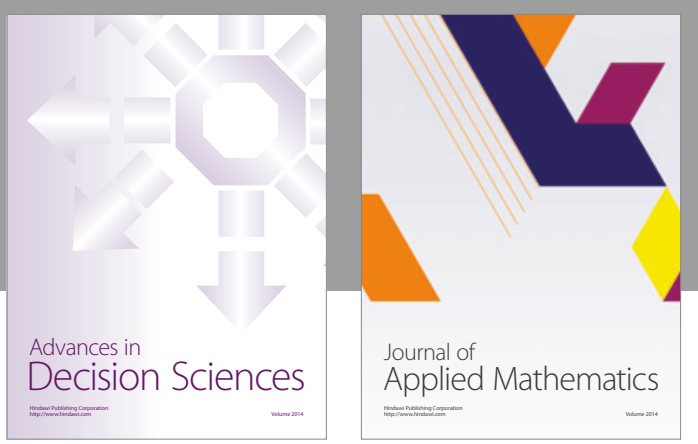

Algebra

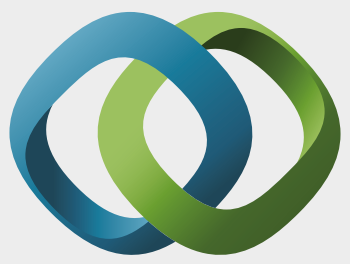

\section{Hindawi}

Submit your manuscripts at

https://www.hindawi.com
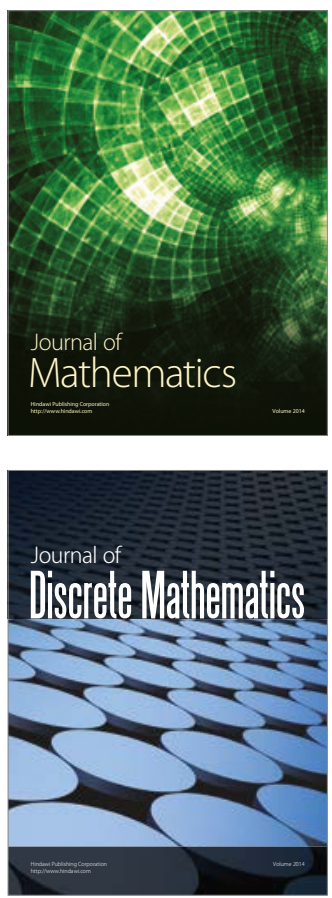

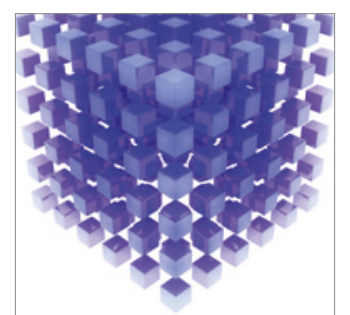

Mathematical Problems in Engineering
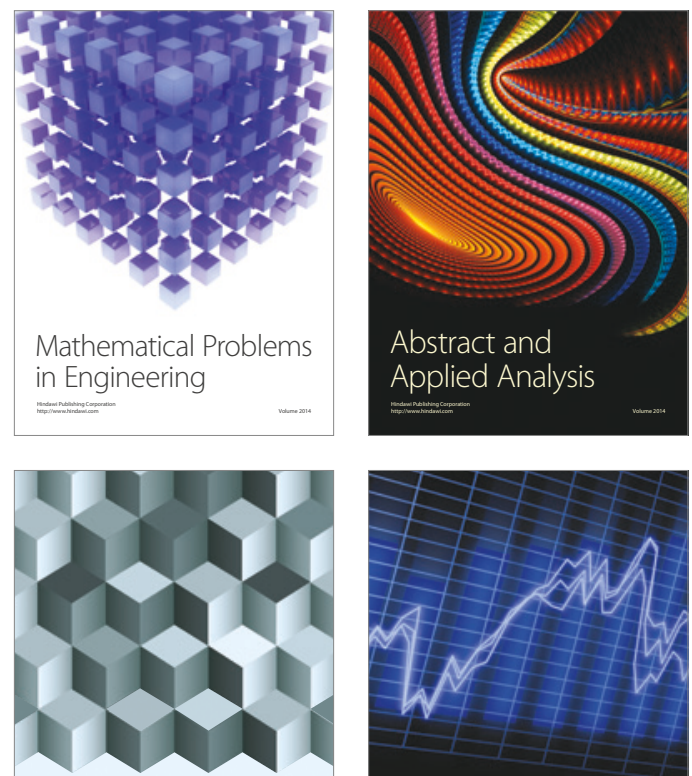

Journal of

Function Spaces

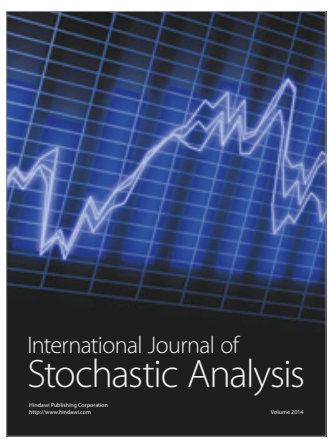

Probability and Statistics
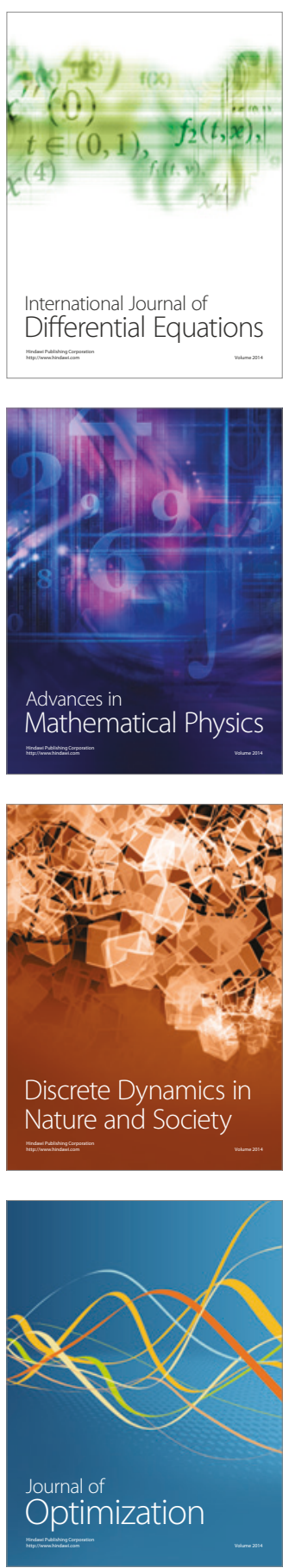